\title{
Optic Flow Estimation using the Hermite Transform
}

\author{
Boris Escalante-Ramírez ${ }^{a}$, Jose Luis Silván-Cárdenas ${ }^{b}$, and Héctor Yuen-Zhou ${ }^{c}$ \\ ${ }^{a}$ boris@servidor.unam.mx \\ bjlsilvan@centrogeo.org.mx \\ ${ }^{c}$ hyuen@super.unam.mx
}

\begin{abstract}
In this paper we present a spatiotemporal energy based method to estimate movement from an image sequence. A directional energy is defined in terms of the Radon projections of the Hermite transform. Radon transform provides a suitable representation for image orientation analysis, while Hermite transform describes image features locally in term of Gaussian derivatives. These operators have been used in computer vision for feature extraction and are relevant in visual system modeling.

A directional response defined from the directional energy is used to estimate local motion as well as to compute a confidence matrix. This matrix provides a confidence measure for our estimate and it is used to propagate the velocity information toward directions with high uncertainty.

With this results, there can be applications ranging from motion compensation, and tracking of moving objects, to segmentation and video compression.

Keywords:Optic flow, Hermite transform, Radon transform, local projections, local orientation, steering functions, image motion.
\end{abstract}

\section{INTRODUCTION}

It is generally acknowledged that visual perception models must involve two major processing stages: 1) initial measurements and 2) high level interpretation.

Such measurement processes should be image-independent and require no previous or concurrent interpretation. Unfortunately, the most relevant features are not known a priori.

However, we know that for image processing purposes, linear operators that exhibit special kind of symmetries related to translation, rotation and magnification are of particular interest.

A family of generic neighborhood operators fulfilling these requirements is that formed by the so-called Gaussian derivatives.

These operators has long been used in computer vision for feature extraction ${ }^{2}$ and are relevant in visual system modeling. ${ }^{21}$

Formal integration of these operators is achieved in the Hermite transform introduced by Martens, ${ }^{13} \cdot{ }^{14}$

This transform can take many alternative forms corresponding to different ways of coding local orientations of an image. ${ }^{15}$ For instance, we show in this paper that the 1-D Hermite coefficients of the 3-D Radon transform ${ }^{12}$ of a localized signal can be expressed in terms of the 3-D Hermite coefficients and some steering functions. Conceptually, local Radon projections provides a suitable representation for image orientation analysis, while Hermite transform describes the image features in term of Gaussian derivatives.

This representation allows, under certain circumstances, to estimate an orientation related to the local motion associated to a spatiotemporal signal.

Because motion estimation is an ill-posed problem, i.e., there is not a unique solution, it is necessary to solve ambiguities by regarding a smoothness constraint on the velocity field. This smoothness constraint must allow

Division de Estudios de Posgrado

Facultad de Ingeniería

Cto Ext S/N UNAM 
field discontinuities along moving edges. The constraint is maximized by using variational calculus which was first proposed by. ${ }^{9}$

Some of the algorithms used to solve this problem are the gradient based methods, ${ }^{9}$ or correlation based ones. ${ }^{16}$ Both have proven to be relatively accurate, and very useful in applications such as data compression, ${ }^{11}$ but in applications such as computer vision, it is required that relative errors to be less than $10 \%$, so other techniques must be explored.

There are several methods which combine traditional algorithms with other techniques. ${ }^{16}$ uses a correlation approach combined with feedback, leading to less errors than using the conventional approach. ${ }^{3}$ combine the gradient-based algorithm with neural networks, leading to satisfactory results when dealing with natural scenes that contain noise such as in TV image sequences.

By using wavelet methods ${ }^{22}$ it has been found that there is a more robust estimation when dealing with large displacements, without blurring images.

\section{NOTATION}

Since we use Gaussian filters for the Hermite transform analysis with identical scale along all dimensions, we have to agree on some equivalence between spatial and temporal dimensions. Therefore, we will map all spatiotemporal signals $l(x, y, t)$ onto 3 -D signals $l(x, y, z)$ by setting $z=u t$. The constant $u$ has dimensions of velocity and it determines the velocity range at which the Hermite transform is most sensitive. Notice that the analysis of the signal along dimension $z$ at scale $\sigma$, corresponds to an analysis in time at scale $\sigma / u$.

To simplify the notation we use $l(r)$ instead of $l(x, y, z)$, with $r=(x, y, z)$. For a three-dimensional expansion we denote the Hermite transform coefficients of orders i,j and $\mathrm{k}$ along the dimensions $\mathrm{x}, \mathrm{y}$ and $\mathrm{z}$ respectively, and the window placed at the origin, as $l_{i, j, k}$. The total order is defined by $i+j+k$. A similar notation is used for two dimensions: $l_{i, j}$ for the coefficients of total order $i+j$ and the window placed at the origin.

\section{LOCAL PROJECTION ANALYSIS}

By using the coefficients of the Hermite transform, obtained from the original signal as explained in Appendix A, we can apply the steering functions to locate the patterns we are interested in.

\subsection{Radon projections of Hermite transform}

Here we will focus our attention on the local orientation analysis of a 3-D signal. Since the problem of estimation is mainly concerned with analysis, we will not pay too much attention in the signal reconstruction process. In order to obtain a one-dimensional local representation of the signal; we project $l_{w}(r)=l(r) w^{2}(r)$ on an axis defined by the vector $\alpha=\left(\alpha_{1}, \alpha_{2}, \alpha_{3}\right)$ which is the set of vectors spanning a unitary sphere in the origin. Using spherical coordinates, we get the set of such projections for all $\{(\theta, \phi) ; \theta \in[0,2 \pi), \phi \in[0, \pi)\}$ which is called the 3-D Radon transform of $l_{w}(r)$, mathematically it is expressed as

$$
h_{w}(s, a)=\int_{R^{3}} l_{w}(\mathbf{r}) \delta(\alpha \cdot \mathbf{r}-s) d(\mathbf{r}
$$

The inversion formula for the three-dimensional Radon transform is given through the three-dimensional projection slice theorem. This theorem states that the one-dimensional Fourier transform of the Radon transform of $l(r)$ is the central slice of the three-dimensional Fourier transform of $l(r)$ in the direction $\alpha$.

Substitution of the $3-\mathrm{D}$ expansion for the local signal $l_{w}(r)$ leads to

$$
h_{w}(s, a)=\sum_{n=0}^{\infty} h_{n}(\alpha) d_{n}(-s)
$$

that is the expansion of the projected signal, where 


$$
h_{n}(\alpha)=\sum_{j=0}^{n} \sum_{i=0}^{j} \psi_{i, j-i, n-j}(\alpha) l_{i, j-i, n-j}
$$

for $n=0,1,2, \ldots, \infty$, are the 1-D steered Hermite coefficients in the direction of $\alpha$. These coefficients are a linear mapping of the 3-D Hermite coefficients by the functions

$$
\psi_{m, n, p}(\alpha)=\sqrt{\frac{(m+n+p) !}{m ! n ! p !}} \alpha_{1}^{m} \alpha_{2}^{n} \alpha_{3}^{p}
$$

for $i=0,1, \ldots, j, j=0,1, \ldots, n$. This follows from the steering property of Gaussian derivatives in 3-D.

Moreover, it can be shown that the steering functions satisfy

$$
\sum_{j=0}^{n} \sum_{i=0}^{j}\left\{\psi_{i, j-i, n-j}(\alpha)\right\}^{2}=1
$$

for all $\alpha$ and $n=0,1, \ldots, \infty$. The derivation of this formula is directly obtained by raising the relation $\alpha_{1}^{2}+\alpha_{2}^{2}+\alpha_{3}^{2}=1$ to the $n-t h$ power and applying the definition of Eq.

\subsection{Motion from local orientation}

It is well known that image motion can be characterized by a spatiotemporal orientation along which the timevarying pattern has no high frequency content.

This arises from the fact that two basic conditions must be true within the spatiotemporal window of observation: both illumination and velocity must be constant. The first assumption is true (or almost true) in most practical situations; the second one, however, can be violated in several ways as, for example, when the window is placed near a boundary where occlusion occurs, because more than one movement is observed.

A constant velocity within the window also means there is no rotation nor accelerative effects, which is not the most common case. These drawbacks can be avoided, at least in theory, by using very narrow windows. There is, however, an unavoidable consequence known as the aperture problem. The aperture problem reflects the ambiguity of determining the true movement of oriented patterns seen through an aperture (or sensed by a local operator).

By virtue of the aperture problem, the best we can do is compute the component of velocity in the direction that is normal to the local orientation and give a confidence measure. This measure can be used subsequently to relax the estimation by a global optimization, as pointed out by many authors.

The directional energy is defined as

$$
E(\alpha)=\sum_{n=1}^{\infty}\left\{h_{n}(\alpha)\right\}^{2}
$$

with $h_{n}$ defined in (3). The maximum is obtained at certain orientation $\alpha=\alpha_{0}$ defined in spherical coordinates $\left(\cos \left(\theta_{0}\right) \sin \left(\phi_{0}\right), \sin \left(\theta_{0}\right) \sin \left(\phi_{0}\right), \cos \left(\phi_{0}\right)\right)$, which can be the optic flow orientation. If $\phi_{0} \neq 0$, then it is is a 1 -D pattern moving with normal velocity $u \tan \left(\phi_{0}+\pi / 2\right)\left(\cos \left(\theta_{0}\right), \sin \left(\theta_{0}\right)\right)$ in the image plane $x y$; otherwise, it is a pure temporary pattern such as flickering on a uniform surface.

In case of having several local maxima, we must discard the one that is most close to purely temporal patterns. In practice, we assume that the pattern is constant if the angle $\phi_{0}$ is less than a threshold $t h_{1}$.

The velocity is estimated from the directional energy distribution, by finding the maximum energy, and a confidence measure from its neighborhood.

Thus we obtain a velocity vector and a confidence measure which are given by the expressions (15) and (16).

We use this information to smooth the velocity field through the smoothing constraint, which is going to be explained in the following section. 


\section{OPTIC FLOW ESTIMATION}

\subsection{Practical Issues}

Since the results derived in previous section are based on the continuous Hermite transform, we have to make an approximation of the analog case by using the coefficients of the discrete Hermite transform. If we choose window length $\mathrm{N}$ for spatial dimensions and a window length $\mathrm{M}$ for temporal dimension, then the velocity parameter is approximated as $u=\sqrt{N / M}$. Only the $\mathrm{AC}$ coefficients up to a given total order $\mathrm{P}$ are computed, so that the P-th order energy

$$
E_{p}=\sum_{n=1}^{P} \sum_{j=0}^{n} \sum_{i=0}^{j} l_{i, j-i, n-j}^{2}
$$

has no null terms, i.e., $P \leq \min (M, N)$.

Another consideration that has to be taken into account is the discretization of the unit sphere defined by $\alpha$. We discretize the space of $\alpha$ by using the angles that define a straight line that contains at least three points of the discrete window domain, this is,

$$
\begin{aligned}
& \theta=\arctan (y / x) \quad x, y=-N / 2, \ldots, N / 2 \\
& \phi=\arctan (r / z) \quad r^{2}=x^{2}+y^{2}, z=0, \ldots, N / 2
\end{aligned}
$$

Note that these angles are only in the unit semi-sphere rather than in the whole one, this is because a change of sign in the vector $\alpha$ produces a change of sign of the steered coefficients of odd order, i.e., $h_{n}(-\alpha)=(-1)^{n} h_{n}(\alpha)$, but the contribution of this term to the directional energy remains the same.

It is necessary to detect a local maxima that corresponds to spatiotemporal patterns, because the AC energy $E_{p}$ as well as the directional energy $E(\alpha)$ are sensitive to noise in regions of low contrast, the estimated optimum orientation (as defined in previous section) can differ too much from its real value. Therefore, it is necessary to introduce a more robust estimator and define an uncertainty measure.

The local maxima that corresponds to spatiotemporal patterns is surrounded by a region where energy is decreasing. Because of the noise, it is necessary to estimate the parameters from this local region. Using empirical methods to determine this, it has been found that with less than a half of the total energy, information is not very important. So a barrier of $-3[d B]$ is set.

To simplify calculations, we suppose that there are very small variations in the values of $\phi$, and because we will focus on direction, we will not pay too much attention to the magnitude of the vectors.

From this regions, the parameters $\theta$ and $\phi$ as well as their confidence measure $\sigma_{\theta}$ can be calculated from the first and second moments of the energy distribution.

Because the parameters are given in spherical coordinates, it is necessary to convert them to rectangular coordinates. This information is obtained in the following way

$$
\begin{aligned}
& \mu_{x}=u \tan \mu_{\phi} \cos \mu_{\theta} \\
& \mu_{y}=u \tan \mu_{\phi} \sin \mu_{\theta} \\
& \sigma_{x}=\mu_{x} \sin \mu_{\theta} \sin \sigma_{\theta} \\
& \sigma_{y}=\mu_{y} \cos \mu_{\theta} \sin \sigma_{\theta} \\
& \operatorname{cov}(x, y)=E\left(\left(x-\mu_{x}\right)\left(y-\mu_{y}\right)\right)=E(x y)-\mu_{x} \mu_{y}
\end{aligned}
$$

After this procedure, the velocity and its covariance matrix are given as 


$$
\begin{gathered}
v=\left[\begin{array}{l}
\mu_{x} \\
\mu_{y}
\end{array}\right] \\
\sigma_{v}^{2}=\left[\begin{array}{cc}
\sigma_{x}^{2} & \operatorname{cov}(x, y) \\
\operatorname{cov}(x, y) & \sigma_{y}^{2}
\end{array}\right]
\end{gathered}
$$

We then proceed to smooth the velocity field obtained by this method through the algorithm proposed in the next section.

\subsection{Field Smoothing}

Because uniform regions does not provide enough information to estimate a velocity vector, it is necessary to include a smoothing constraint in our formulation. Field smoothing consists on propagating the velocity information of a given position to all points in its neighborhood. In most works devoted to solve his problem, the smoothing constraint is expressed as a global minimization of the field gradient. In the framework of Hermite transform, field smoothing can be formulated as a minimization of the high order energy of the velocity components. This formulation arises directly from the fact that in regions with uniform motion, the velocity field can be represented by its zero order coefficient for all points. It is however desired the first order energy, which contains most of the information for oriented patterns, not to be minimized near motion boundaries. This is achieved by minimizing the weighted square error

$$
\int_{S_{2}}\left(v-v_{0,0}\right)^{T} E_{1}^{-1}\left(v-v_{0,0}\right) d A
$$

where

$$
E_{1}=\left[\begin{array}{cc}
v_{x_{1,0}}^{2}+v_{x_{0,1}}^{2} & v_{x_{1,0}} v_{y_{1,0}}+v_{x_{0,1}} v_{y_{0,1}} \\
v_{x_{1,0}} v_{y_{1,0}}+v_{x_{0,1}} v_{y_{0,1}} & v_{y_{1,0}}^{2}+v_{y_{0,1}}^{2}
\end{array}\right]
$$

contains the information of the first order energy of $v=\left[v_{x}, v_{y}\right]^{T} . v_{x_{m, n-m}}$ and $v_{y_{m, n-m}}$ denote $n, m-t h$ order coefficients of the 2-D expansion for the velocity vector v. Minimization of 10 plus 11 is possible only approximately by u using the Gauss Seidel relaxation method. The solution is expressed as

$$
\begin{aligned}
v^{0} & =u \\
v^{k+1} & =\left(E_{1}^{-1}+U^{-1}\right)^{-1}\left(E_{1}^{-1} v_{0,0}^{k}+U^{-1} u\right)
\end{aligned}
$$

This expression means that at iteration $k$, the velocity is approximated by the fusion of the zero order coefficient of estimation at iteration $k-1$ and the local estimation.

\subsection{Method description}

The proposed method can be summarized in the following steps (see Figure (1)).

1. Compute the 3-D Hermite transform of the spatiotemporal luminance data. Continuous coefficients are approximated by the discrete Hermite transform using a fast algorithm. In this step we choose the sampling parameter $T \leq N+1$. Of course one would desire a sampling interval $T=N+1$ to reduce computation time. Unfortunately step four requires a small $T$, ideally $T=1$.

2. Steer the Hermite coefficients. We compute the steered Hermite coefficients by applying formula 5

3. Estimate the local motion

4. Propagate velocity information. Compute the velocity vector iteratively with equation 12 . Iterate until changes between successive iterations is below a predefined tolerance. 


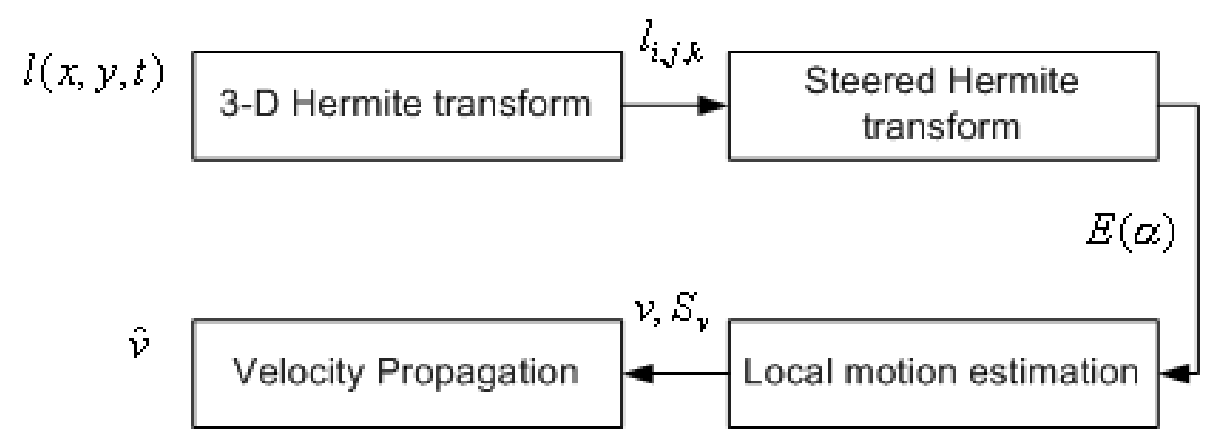

Figure 1. Optic Flow Estimation Algorithm

\section{EXPERIMENTAL RESULTS}

In the following figures, we are going to show some of our experimental results for estimating the optic flow. We are going to analyze some synthetic sequences and a real one. In the synthetic sequences, we are also going to illustrate energy distributions for some of the most common cases.

\subsection{Texture Secuence}

In figure (2) synthetic texture is shown, and was animated using the flow field in figure (3a). The local motion recovered from the directional response obtained after velocity propagation is shown in (3b).

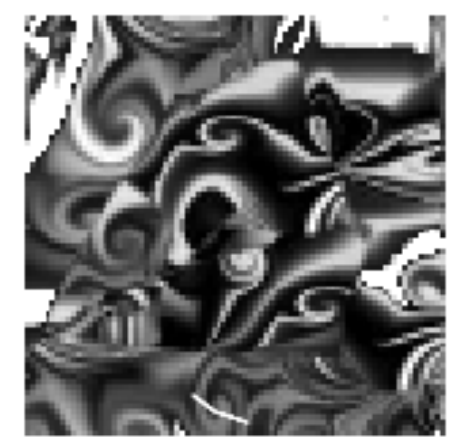

Figure 2. Texture image

\subsection{INTERVIEW Sequence}

We use the INTERVIEW sequence to illustrate some of the most common energy distributions that we can find in sequences. The complete frame is shown in figure (4), and some amplified parts are shown in the next figures.

In figure (5) we can find a purely spatial feature, this is, a noticeable change in brightness between two regions, and although there is real motion, the algorithm cannot see it because of the aperture problem. That is why the energy distribution has lobules pointing inside the xy plane.

In contrast, figure (6) is a pattern in which the temporal component is predominant over the spatial one. We can see the lobules getting out of the $x y$ plane.

And finally, in figure (7), the spatial and temporal components are comparable, so the lobules of the energy distribution are inclined and the componenwe cannot see the energy inclined. 


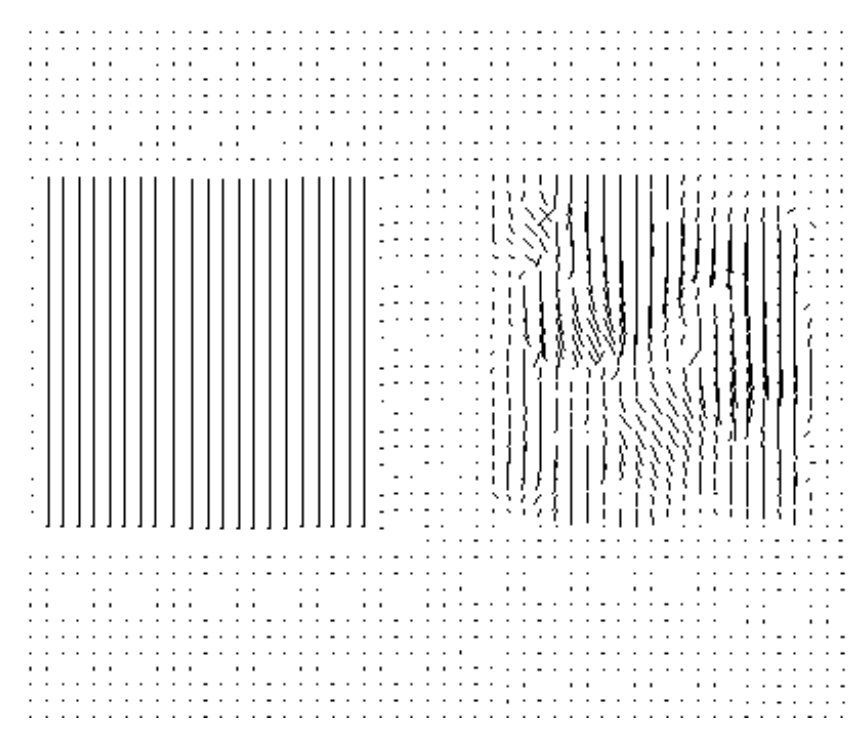

Figure 3. a)Original Flow b)Estimated Flow

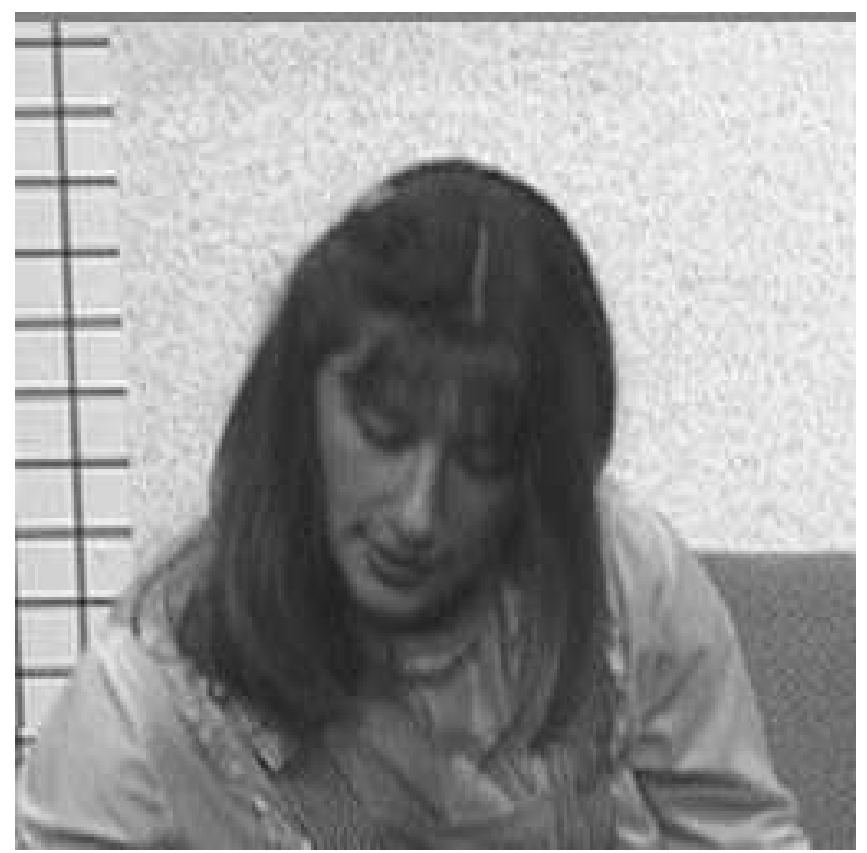

Figure 4. Interview Sequence

\subsection{EcoSonar Heart Sequence}

In figure (8) there is a sonar heart image and in figure the optic flow estimated by the algorithm. We can use this results to aid physicians when diagnosing a disease. Conventional X-rays do not show movement, and video equipment can be very expensive and difficult to transport from one place to another. In this case we can use optic flow to give an initial idea of what is happening, and in case of abnormal patterns, a more detailed diagnosis should be done. 


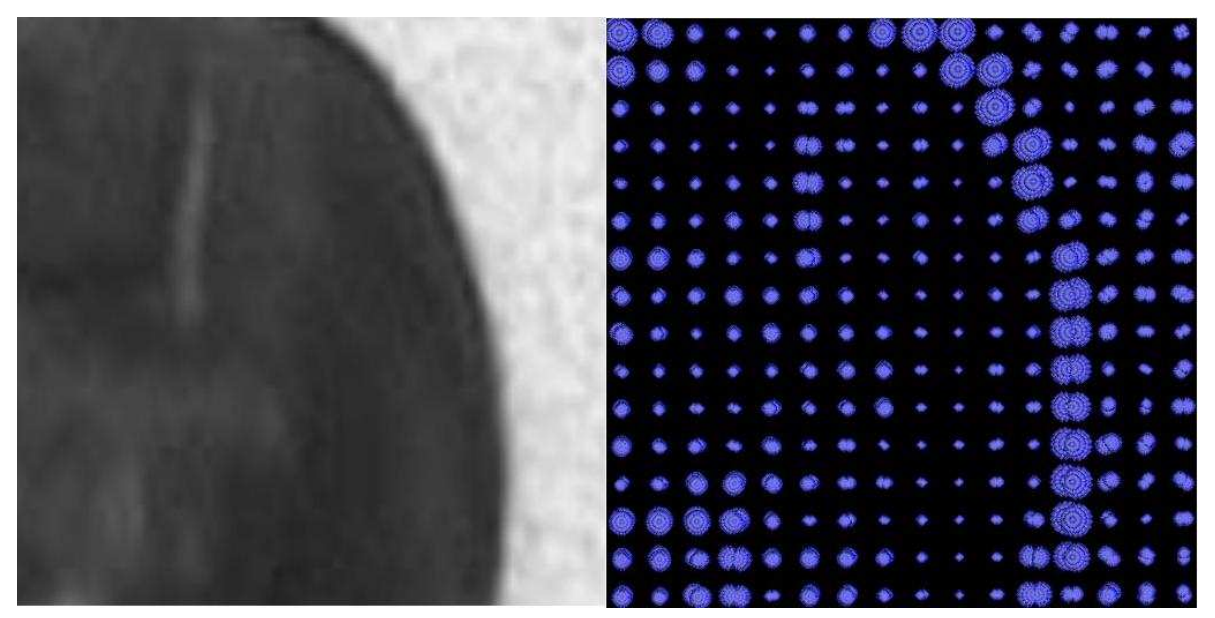

Figure 5. Purely Spatial Feature

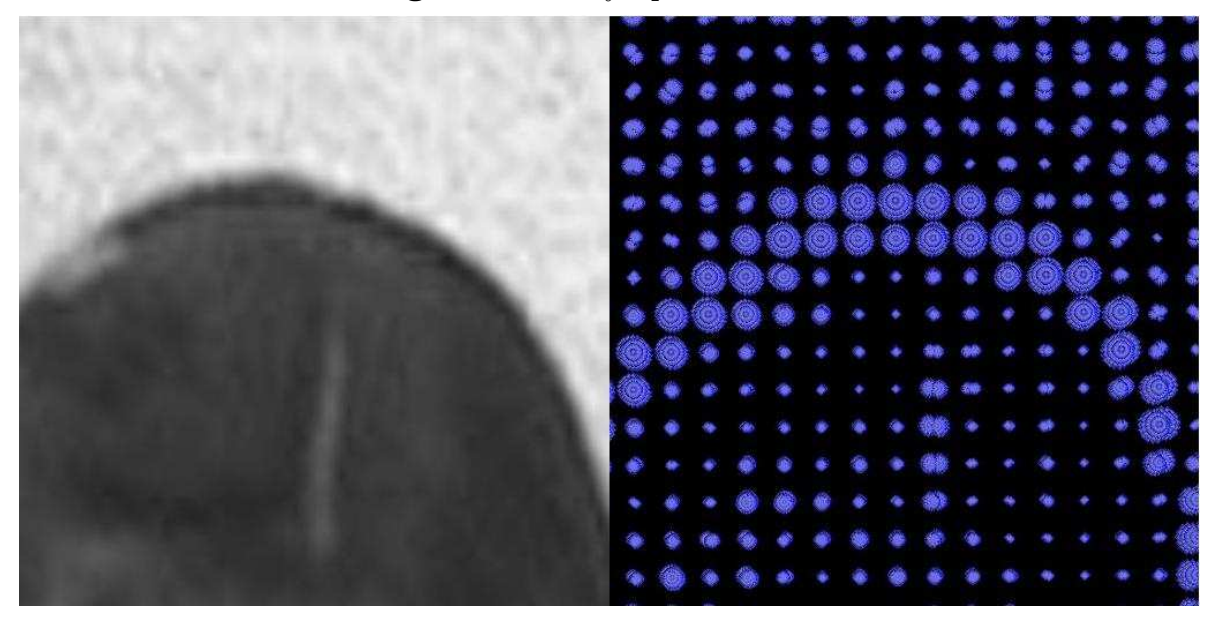

Figure 6. Purely Temporal Feature

\section{CONCLUSIONS}

In this work, an alternative algorithm for optic flow estimation is proposed. This method is based on the psycophysical model of the human vision, which can give more information than traditional methods in some cases.

The energy distributions obtained by steering the Hermite transform coefficients can be used to identify many phenomena, such as purely spatial patterns, purely temporal patterns, or a mixture of this two phenomena, leading to spatiotemporal energy.

The use of this energy distributions can help us to differentiate between temporal patterns, which are our main concern, from spatial ones, and are found to be relatively accurrate when looking for displacement.

We use this patterns to determine the optic flow where the aperture problem is not present, and then the information propagation is done by the technique proposed in this document.

The use of this algorithm in real sequences such as medical images where there are noisy environments can be useful for aiding physicians to detect anomalies in their patients. 


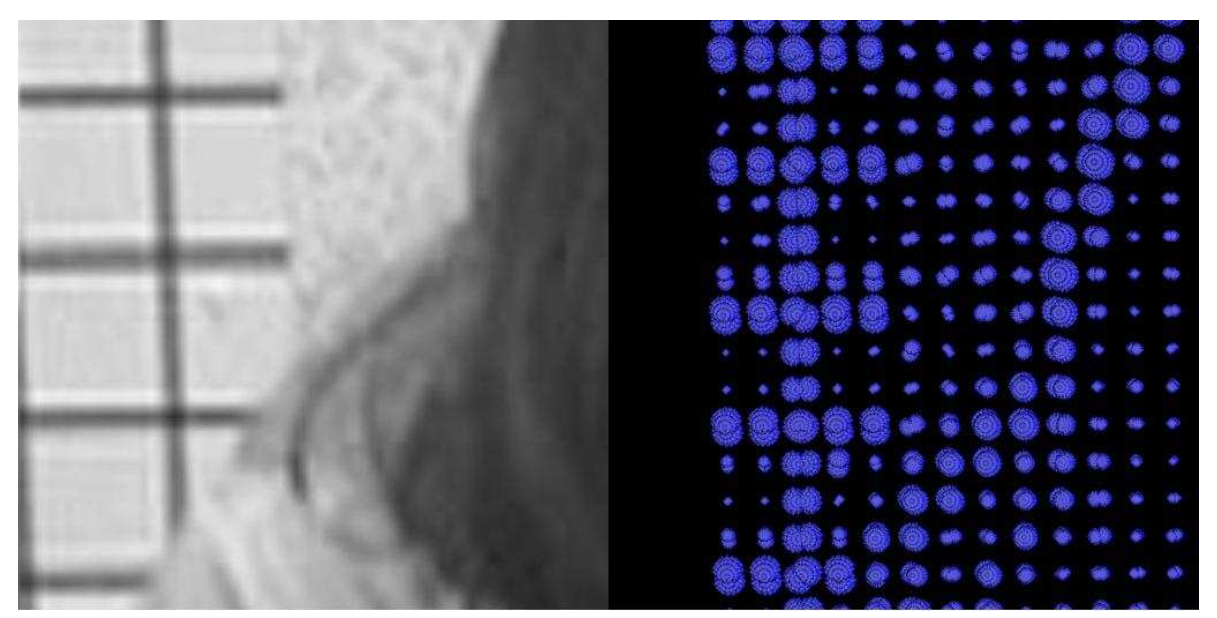

Figure 7. Spatial and Temporal Feature

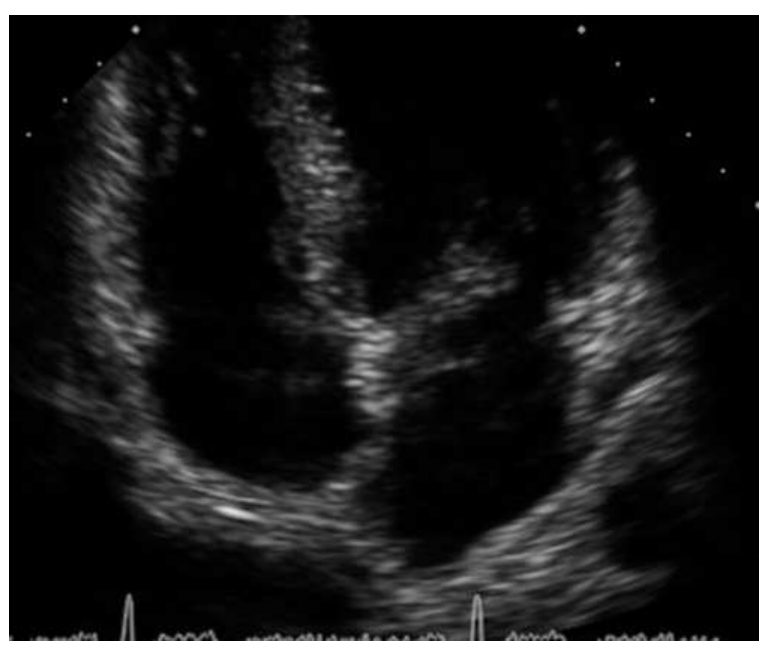

Original Image

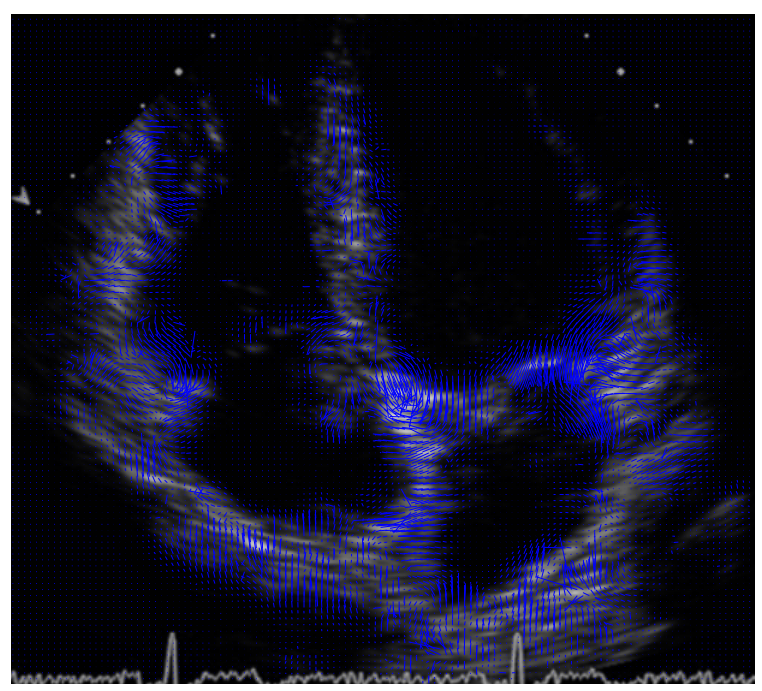

Optic Flow

Figure 8. EcoSonar Heart Sequence

\section{APPENDIX A. THE HERMITE TRANSFORM}

This transform is a particular case of a family of transformations known as polynomial transforms, which are determined by the window function $w(x)$. For the case we are interested in, the window corresponds to a Gaussian function, defined in one dimension by

$$
w^{2}(x)=\frac{1}{\sigma \sqrt{\pi}} e^{-(x / \sigma)^{2}}
$$

with the scale parameter $\sigma>0$. We are going to define the concepts in one dimension, which can be extended to multiple dimensions straightforward.

In the analysis stage, the input signal $l(x)$ is firstly localized by multiplying it by the window $w^{2}(x-m T)$ at positions $m T$, for $m \in Z$ and $T>0$. Then, the portion of the signal within the window, is expanded on the basis $g_{n}(x-m T) w^{2}(x-m T), n=0,1,2, \ldots, \infty ; m \in Z$ with 


$$
g_{n}(x)=\frac{1}{\sqrt{2^{n} n !}} H_{n}(x / \sigma)
$$

where $H_{n}(x)$ denotes the Hermite polynomial of order $n$. The expansion is expressed under general conditions as

$$
w^{2}(x-m T)\left[l(x)-\sum_{n=0}^{\infty} l_{n}(m) g_{n}(x-m T)\right]=0
$$

where the Hermite coefficients $l_{n}[m]$, for $n=0,1,2, \ldots$ and $m \in \mathbf{Z}$ are given by

$$
l_{n}(m)=\int_{-\infty}^{\infty} l(x) g_{n}(x-m T) w^{2}(x-m T) d x
$$

which is equivalent to sample the output of the convolution between the signal and the decimation filters $d_{n}(x)=g_{n}(-x) w^{2}(x)$, for $n=0,1,2, \ldots$ at points $m T$, for $m \in \mathbf{Z}$. The impulse response of these filters corresponds, except for a constant, to Gaussian derivatives, since they can be written as

$$
d_{n}(x)=\frac{1}{\sqrt{2^{n} n !}} \frac{d^{n}}{d(x / \sigma)^{n}} w^{2}(x)
$$

Therefore, the Hermite transform describes the input signal $l(x)$ in terms of Gaussian derivatives at a given scale for all positions belonging to the sampling lattice $S=\{m T ; m \in \mathbf{Z}, T>0\}$. While the signal $l(x)$ is synthesized from the coefficients $l_{n}[m]$ by integrating the local expansions of (1), provided that the weighting function

$$
W(x)=\sum_{m=-\infty}^{\infty} w^{2}(x-m T)
$$

is different from zero for all $x$, this is

$$
l(x)=\sum_{n=0}^{\infty} \sum_{m=-\infty}^{\infty} l_{n}(m) s_{n}(x-m T)
$$

with the interpolating filters

$$
s_{n}(x)=g_{n}(x) \frac{w^{2}(x)}{W(x)}, n=0,1,2, \ldots, \infty
$$

The local signal

$$
l_{w}(x-m T)=w^{2}(x-m T) l(x)=\sum_{n=0}^{\infty} l_{n}(m) d_{n}(m T-x)
$$

expresses the contribution of the coefficients $l_{n}(m), n=0,1,2, \ldots$ at positions $m T$ over all the synthesized signal.

For a D-dimensional input signal we apply the same analysis and synthesis stages for each dimension, since a D-dimensional (isotropic) Gaussian windows is defined by multiplying D one-dimensional Gaussian Windows with the same scale parameter $\sigma$. 


\section{A.1. Discrete Hermite Transform}

The discrete equivalent of the Hermite transform is based on the approximation of the Gaussian window by a binomial window

$$
w^{2}[x]=\frac{1}{2^{n}}\left(\begin{array}{l}
N \\
x
\end{array}\right)
$$

for $x=0, \ldots, N$. The polynomials that are orthonormal respect to this window are the Krowtchouk polynomials, then

$$
g_{n}[x]=\frac{1}{\sqrt{\left(\begin{array}{l}
N \\
n
\end{array}\right)}} \sum_{k=0}^{n}(-1)^{n-k}\left(\begin{array}{c}
N-x \\
n-k
\end{array}\right)\left(\begin{array}{l}
x \\
k
\end{array}\right)
$$

There is an asymptotic approximation between these discrete function and the corresponding continuous ones, for $N \infty$, i.e.,

$$
\begin{aligned}
& w^{2}[x-N / 2]=\frac{1}{\sqrt{\pi N / 2}} e^{-(x / \sqrt{N / 2})^{2}} \\
& g_{n}[x-N / 2]=\frac{1}{\sqrt{2^{n} n !}} H_{n}(x / \sqrt{N / 2})
\end{aligned}
$$

Hence, the discrete Hermite transform of length $N$ approximates the continuous Hermite transform of scale $\sigma=\sqrt{N / 2}$. The properties of the discrete Hermite transform can therefore be predicted quite accurately from the corresponding properties of the continuous Hermite transform.

The decimation and interpolation filters are centered on the origin by shifting the window over $N / 2$, which leads to the following definition for the decimation filter. These functions can be also expressed as

$$
d_{n}[N / 2-x]=\frac{(-1)^{n}}{\sqrt{\left(\begin{array}{c}
N \\
n
\end{array}\right)}} \Delta^{n}\left\{\left(\begin{array}{l}
x \\
n
\end{array}\right) w^{2}[x]\right\}
$$

where $\Delta^{n}$ is the nth order forward difference operator. These filters can be efficiently implemented by applying the kernel $\{1,1\}, N-n$ times and the kernel $\{-1,1\}, n$ times, for $n=0,1, \ldots, N$ and normalizing the output.

\section{REFERENCES}

1. Adelson, E. H. and Bergen, J. R., Spatiotemporal energy models for the perception of motion, Journal of the Optical Society of America A, 2:284-299, 1985.

2. Bevington, J. and Mersereau, R., Diferential operator based edge and line detection In Proc. ICASSP, 249-252, 1987.

3. Brattoli, M.; Convertino, G.; Distante, A.; Branca, A., A Neural Network computing Observerś heading from Optic Flow, Neural Networks, 1994. IEEE World Cong. on Comp. Intell., Vol 7 , 4359-4364, July 1994.

4. Cheng C.C., Ho K.H., Li H.T., Lin G.L., Image Following using the Freature-Based Optic Flow approach, Proceedings of the 2002 IEEE, ISIC, 2002.

5. Fleet, D. J. and Jepson, A. D., Hierarchical construction of orientation and velocity selective filters. IEEE Trans. on Pattern Analysis and Machine Intelligence 11(3): 315-325, 1989.

6. Ghosal, S.; Mehrotra, R., Robust Optical Flow Estimation, Image Processing, 1994. Proceedings. ICIP-94., IEEE Intl. Conf., Vol 2 , 780-784, Nov. 1994.

7. Heeger, D. J., A model for the extraction of image flow, Journal of the Optical Society of America A, 4:1455$1471,1987$. 
8. Hiraiwa, A.; Fuse, K.; Komatsu, N.; Komiya, K.; Ikeda, H., Accurate estimation of optical flow for fully automated tracking of moving-objects within video streams, Circ. and Syst., ISCAS '99. IEEE ,Vol 4, 515519, June 1999.

9. Horn, B. K. P. and Schunck B.G., Determining the optical flow, Artificial Intelligence 17. pp 185-203, 1981.

10. Huang, Y.; Palaniappan, K.; Zhuang, X.; Cavanaugh, J.E., Optic Flow Field seg. and Motion Est. Using a Robust Genetic Partitioning Alg., Patt. Anal. and Mach. Intell., IEEE Trans. on ,Vol 17 , Iss 12, 1177-1190, Dec. 1995.

11. Lin S., Shi Y.Q., An optical flow based motion compensation algorithm for very low bit-rate video coding, IEEE 1997.

12. Ludwig, D. The Radon transform on Euclidian Space. Commun. Pure Apppl. Math. 49-81, 19 (1966).

13. Martens, J. B., The Hermite Transform theory. IEEE Trans Acoust Speech Signal Processing 38(9): 15951606, 1990a.

14. Martens, J. B., The Hermite Transform applications. IEEE Trans Acoust Speech Signal Processing 38(9): 1607-1618, 1990b.

15. Martens, J. B., Local Orientation Analysis in Images by means of Hermite Transform. IEEE Trans on image proccesing 6(8): 1595-1606, 1997.

16. Pan, J.N.; Shi, Y.Q.; Shu, C.Q., Correlation-feedback Approach to Computation of Optical Flow, Circ. and Syst., 1994. ISCAS '94., 1994 IEEE, Volume: 3 , 33-36, June 1994.

17. Silván-Crdenas, J. L. and Escalante-Ramírez, B. Polynomial transform application to motion estimation, Mathematical modeling and estimation techniques in Computer Vision (E. Dougherty, J.L. Davison, T. Petreux, eds.) en Proc. of SPIE. vol. 3457, 1998.

18. Singh, A., Optic Flow Computation: A Unified Perspective. IEEE Computer Society Press. Los Alamitos, Cal, 1991.

19. Szeg, G., Orthogonal Polynomials, American Mathematical Society. Colloquium Publications, 1959.

20. Watson, A. B., and Ahamuda, A. J., Model of human vision motion sensing, Journal of the Optical Society of America A, 2:322-342, 1985 .

21. Young, R., The Gaussian derivative theory of spatial vision: Analysis of cortical cell receptive field lineweighting profiles. General Motors Res. Labs., Rep. 4920, 1986.

22. Yu-Te Wu; Kanade, T.; Cohn, J.; Ching-Chung Li, Optical Flow Estimation using Wavelet Motion Model, Computer Vision, 1998. Sixth International Conference on , 992-998, 4-7 Jan. 1998. 